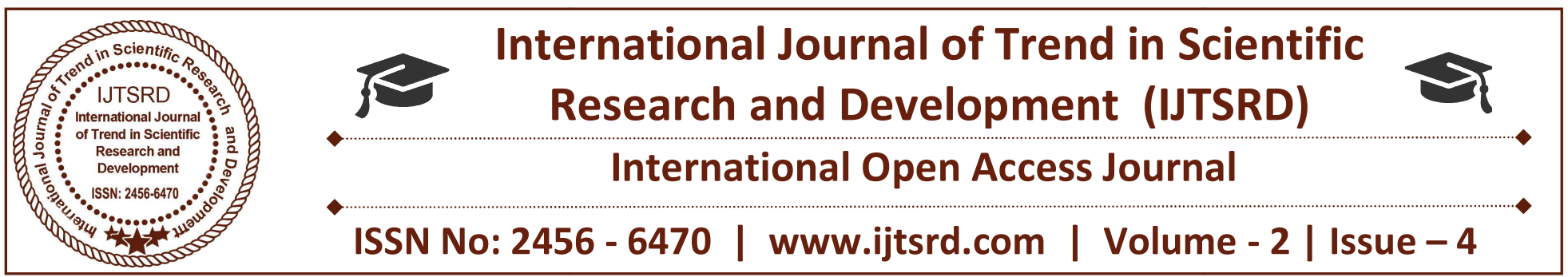

\title{
Crack Detection in Ceramic Tiles using Zoning and Edge Detection Methods
}

\author{
Bhagyashree R K $\mathbf{1}^{1}$, S. A. Angadi ${ }^{2}$ \\ ${ }^{1}$ M. Tech Scholar ${ }^{2}$ Professor \\ Department of CSE, Visvesvaraya Technological University, Belagavi, Karnataka, India
}

\begin{abstract}
The quality control process in ceramic tile industry plays crucial role to enhance the quality standards. At present the quality analysis is mostly done manually. Manual inspection is not so efficient and it is labour intensive. Defect detection accuracy is lower due to human mistakes and unforgiving mechanical condition. To vanquish these issues, an automated inspection system for crack tiles that depends on image processing methods is presented. The tiles are examined using image processing concept using matlab software. The processing is very less compared to that of manual inspection. The automated inspection system can replace the manual ceramic tile detection system more efficiently and with better accuracy.
\end{abstract}

Keywords: tiles defect, Image pre-processing, binary morphology, sub-blocking, binarization, crack detection.

\section{INTRODUCTION}

In modern world, almost every industry is automated to achieve the comparative advantages over manual operations. The manufacturing process in ceramic tiles is mostly automated except the final process of inspection. The inspection stage is very critical to maintain the quality and the customer satisfaction, but still done manually.

Computer technology has become the fastest and rapidly growing field in the past couple of decades as it has outstanding advantages of high accuracy rate, high reliability and high user friendliness.
Manual inspection system is based on the human judgment. The quality of human judgment depends on the prior knowledge, training and experience of the inspecting of the employee. The main disadvantage of the manual inspection is that the decision made on the defect detection may vary on the person operating. Further the inspection for long period of time may cause eye fatigue and the brain gets tired and this may lead to wrong inspections or decision makings.

The rest of paper is organized as follows. Section 2 describes the related work. Section 3 presents proposed methods. Section 4, shows experimental results. Section 5 concludes the paper.

\section{RELATED WORK}

In paper, H. Elbehiery et al. have proposed an algorithm for surface defect detection on ceramic tiles which is based on Image processing and morphological techniques [1]. First part of the algorithm starts with image acquisition, and then histogram equalization is performed on the images.

In second paper,[2], Boukouvalas et al. applied techniques based on a set of separable line filters, through textured tile crack detector based on the Wigner distribution and a conjoint spatial frequency representation of texture,

In third paper, Yiyang et al. [3] have proposed a crack detection algorithm based on digital image processing technology. By pre-processing, image segmentation and feature extraction, they have obtained the information about the crack image 
In fourth paper [4], Alam et al. have proposed a detection technique by the combination of the digital image correlation and acoustic emission. The former method gives a very precise measurement of surface displacements, thus crack openings and crack spacing were determined.

In referred paper used the co-occurrence matrix in order to extract the features of the ceramic tiles. This is method have been applied to texture classification of aerial photographs and photomicrographs [5].

\section{PROPOSED METHODOLOGY}

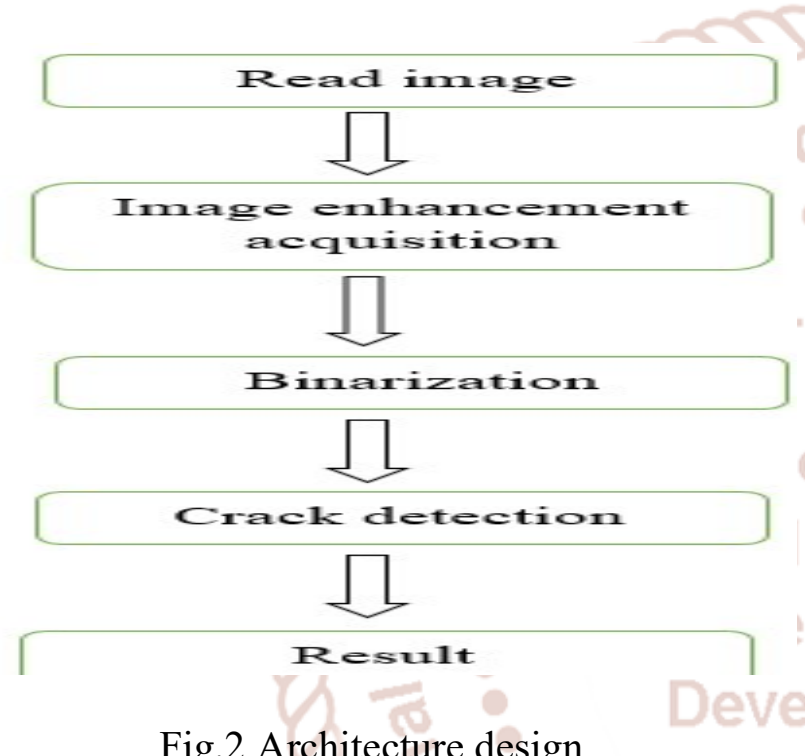

Fig.2 Architecture design

Pre-processing: The input is taken has the image of the cracked tile. In this step, it remove if their present any external noise in image. The main idea is to find the clear image so that it can be further processed. Then it is converted into grayscale from the color image, which is easy for the extraction. By using the edge detector contour of the tile is identified.hsv = rgb2hsv(rgb) changes over RGB esteems to the fitting tone, immersion, and esteem (HSV) arranges. rgb can be a p-by-3 colormap cluster or a m-by-n-by-3 picture exhibit. hsv is an indistinguishable size from rgb.

The result of applying an edge detection to an image may prompt an arrangement of associated curves that show the limits of items, the limits of surface markings and in addition curves that compare to discontinuities in surface orientation. Along these lines, applying an edge recognition calculation to a picture may essentially decrease the measure of information to be handled and may consequently reduce amount of data that might be viewed as less relevant, while saving the critical basic properties of a picture. In the event that the edge detection step is fruitful, subsequent task of interpreting the information contents in the original picture may accordingly be generously streamlined. In any case, it isn't generally conceivable to acquire such perfect edges from genuine pictures of moderate complexity.

Image enhancement is done, the normal image that is given as input is converted into binary image as the result has to be in $0 \mathrm{~s}$ and $1 \mathrm{~s}$. This method of converting image to binary image is known as binarization. In the enhancement part, the image is filtered and the crack is marked. Binary morphology method is applied.

IM2 = imerode (IM,SE) dissolves the grayscale, twofold, or pressed double picture IM, restoring the disintegrated picture, IM2. With is help of sub blocking the image is marked in blocks. The image is given a particular threshold to identify the crack in the input image. Where ever the black pixel in found, it marks it as 1 , the remaining is shown as 0 .

\section{RESULTS}

The ceramic tile image is converted to binary digits and then checked horizontally line by line for the crack in the image.

Below images show the process carried out.

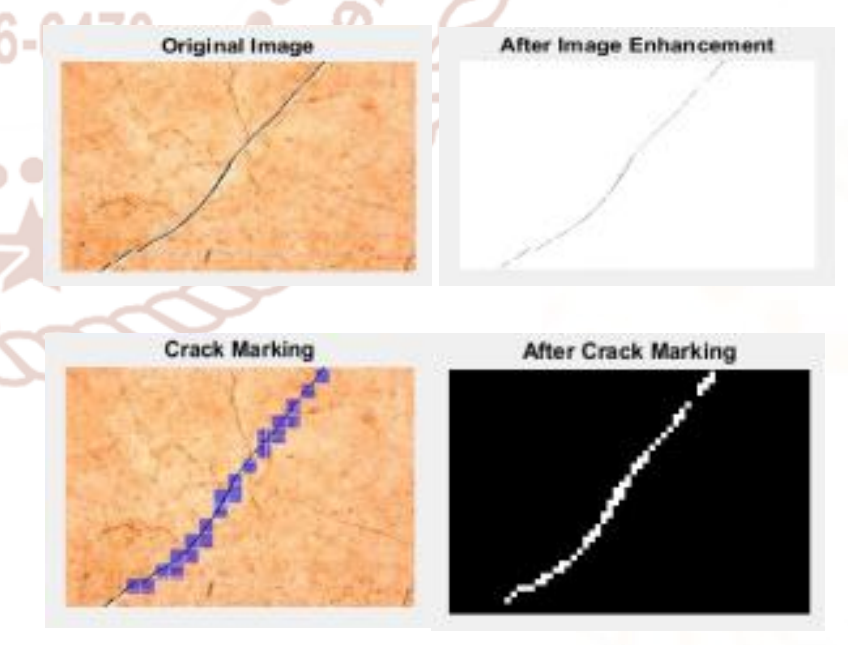

The image is enhanced and then the crack is detected. Same procedure is carried out for different types of cracked tiles. Then the accuracy is calculated for the proposed framework to check whether it gives better accuracy in short time.

ROC curve, is a graphical plot that outlines the analytic capacity of a binary classifier framework as 
its threshold is varied. The ROC curve is made by plotting the true positive rate (TPR) against the false positive rate (FPR) at different threshold set. The true positive rate is otherwise called affectability, review or likelihood of detection[1] in machine learning. The false-positive rate is otherwise called the drop out or likelihood of false alarm[1] and can be computed as (1- specificity).

When all is said in done, if the probability distributions for both detection and false alert are known, the ROC curve can be created by plotting the cumulative distribution function of the identification probability in the y-pivot versus the cumulative distribution function of the false-caution probability on the x-pivot. The ROC is otherwise called a relative operating characteristic curve, since it is a correlation of two working qualities (TP and FP) as the criterion changes.

\begin{tabular}{|c|c|c|}
\hline Actual type & Predicted type & Result status \\
\hline Crack (37) & Crack (36) & TP (97.2) \\
\hline Crack (37) & No crack (1) & FP (2.7) \\
\hline No crack (16) & No crack (15) & TN (93.7) \\
\hline No crack (16) & Crack (1) & FN (6.2) \\
\hline
\end{tabular}

The accuracy formula to find the goodness of the proposed framework

Accuracy (ACC) $=\Sigma$ True positive (TP) $+\Sigma$ True negative (TN) $/ \Sigma$ Total population

The proposed system is tested on total 53 images to check the correctness of the system.

$\mathrm{TP}=36$

$\mathrm{TN}=15$

We have used ROC Curves to find the goodness of the accuracy.

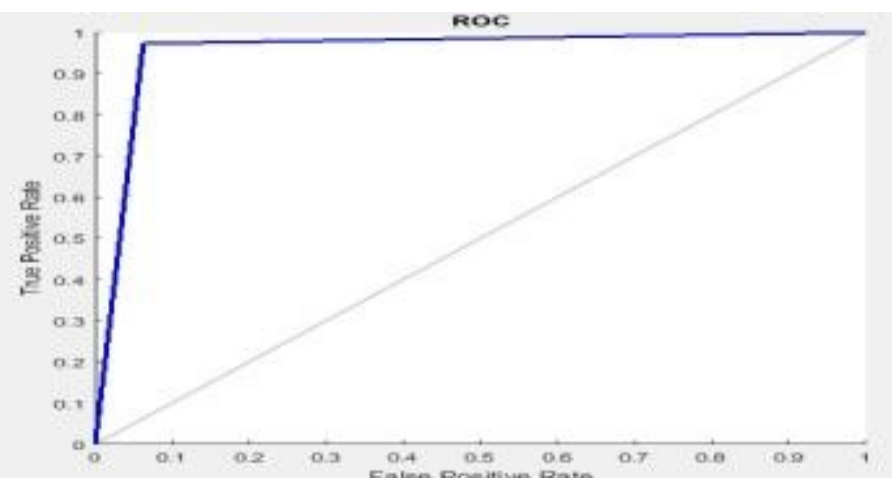

The dark line shown above in the graph represents the accuracy of the proposed framework. Here the accuracy rate is $96.23 \%$.

\section{CONCLUSION AND FUTURE SCOPE}

This work presents a complete system for automatic crack detection of ceramic tiles using image processing and machine learning.

This system can be used to the current market ceramic tile industry as substitution to the manual inspection system to achieve high accuracy, efficiency and to overcome the other drawbacks of manual inspection system.

In future we can make robust framework with dataset where all types of ceramic tiles are stored and cracks are detected.

\section{REFERENCES}

1. Boukouvalas C., Kittler J. , Marik R., Mirmehdi M., and Petrou M.,2006, "Earthenware tile examination for shading and basic defects", project no. BE5638, pp 6, College of Surrey.

2. Elbehiery H., Hefnawy An., and Elewa M., 2005, "Surface imperfections identification for earthenware tiles utilizing picture preparing and morphological techniques", world institute of science, engg and tech.

3. Merazi-Meksen, 2016 "Utilization of an eyetracker to survey laborers in earthenware tile surface deformity identification" $<\mathrm{em}>\mathrm{St}$. Julian's $</$ em $>$ pp. 088-091.

4. R. Gonydjaja Bertalya Kusuma, 2014 "Rectangularity Imperfection Identification for Earthenware Tile Utilizing Morphological Procedures" <em $>$ ARPN Diary of Designing and Connected Sciences $</$ em $>$ pp. 2052-2056.

5. A. Agarwal K. Goel , 2016 "Relative Examination of Advanced Picture for Edge Discovery by Utilizing Bacterial Scrounging and Shrewd Edge Identifier" <em> Second Worldwide Meeting on Computational Knowledge and Correspondence Innovation $(\mathrm{CICT})</ \mathrm{em}>$ pp. 125-129 2016.

6. Ž. Hocenski, T. Keser, 27 July 2007 Disappointment discovery and seclusion in clay tile edges in light of form descriptor investigation. In Procedures of the fifteenth Mediterranean Meeting on Control and Robotization, pp. 1-6. 
International Journal of Trend in Scientific Research and Development (IJTSRD) ISSN: 2456-6470

7. National Instruments, June 2009 NI, ni.com, and Lab VIEW are trademarks of National Instruments Organization. Allude to the Outline of the PID and Fluffy Rationale Toolbox, Section Number 351286B-01

8. Meenakshi, Gurleen , 2012 "Classifier examination for imperfection recognition in earthenware tiles utilizing glcm", IJLTEMAS VOLUME I ISSUE VII 2ICAE GOA, ISSN 22782540 .
9. Liujie , 2006 "SVM-KNN Discriminative Closest Neighbor Grouping for Visual Class Acknowledgment", Continuing of the IEEE (CVPR'06).

10. D. O. Aborisade, s2008 "PC vision framework for programmed surface review of plain fired divider tile" Diary of Building and connected sciences $3(11): 865-871$

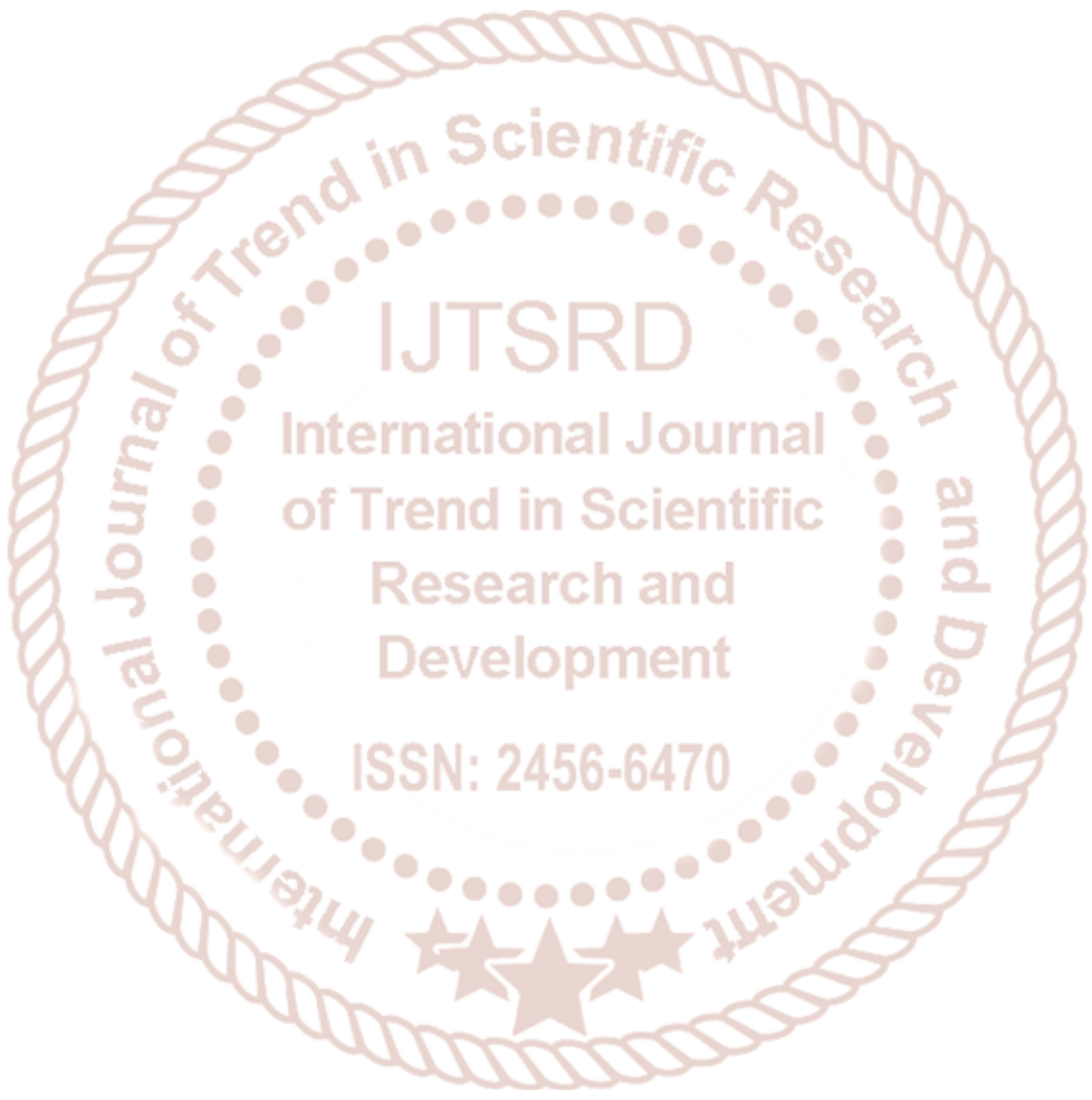

